ORIGINAL ARTICLE

\title{
Low grade malignant peripheral nerve sheath tumour: varied cytological and histological patterns
}

\author{
U Yamaguchi, T Hasegawa, T Hirose, H Chuman, A Kawai, Y Ito, Y Beppu
}

J Clin Pathol 2003;56:826-830

See end of article for authors' affiliations .....................

Correspondence to: Dr T Hasegawa, Pathology Division, National Cancer Centre Research Institute, 5-1-1 Tsukiii, Chuo-ku, Tokyo 104-0045, Japan; tdhasega@ncc.go.jp

Accepted for publication 14 May 2003
Background: A small number of malignant peripheral nerve sheath tumours (MPNSTs) are low grade, and the nature of these low grade tumours has never been systematically assessed.

Aims: To describe the clinicopathological, immunohistochemical, and ultrastructural features of low grade MPNST and to discuss the main differential diagnoses.

Methods: Four cases of low grade MPNST were studied, including one coexistent with neurofibromatosis type 1. The tumours were analysed with respect to nuclear atypia, cellularity, nuclear enlargement, hyperchromasia, mitotic rate, and necrosis. Immunohistochemistry was performed by standard techniques, and an ultrastructural study was performed on one tumour.

Results: The ages of the patients ranged from 32 to 72 years (mean, 58). Two were male and two were female. Three tumours occurred in the deep tissue, including one in the retroperitoneum, and one was located in the dermal and subcutaneous tissue. The maximum diameters of the tumours ranged from 3.5 to $8.0 \mathrm{~cm}$. Microscopically, all tumours showed moderate hypercellularity, an increased nuclear to cytoplasmic ratio, and hyperchromasia, but exhibited varied growth patterns, including those that were atypical neurofibroma-like, low grade fibromyxoid sarcoma-like, low grade epithelioid, and haemangiopericytoma-like. All tumours showed immunoreactivity for S-100 protein and vimentin.

Conclusions: These findings suggest that careful clinical and histological evaluation, along with S-100 protein immunostaining, are essential for the accurate diagnosis of low grade MPNST.
$M$ alignant peripheral nerve sheath tumours (MPNSTs) arise from a peripheral nerve or show intrinsic nerve sheath differentiation. They comprise approximately $5 \%$ of all malignant soft tissue tumours. ${ }^{1}$ Most are derived from neurofibromas, often of the plexiform type or neurofibromatosis type 1 (NF1), or arise de novo in normal peripheral nerves. $^{23}$

"It is usually difficult to distinguish neurofibromas with varying degrees of atypia from malignant peripheral nerve sheath tumour"

The histological features of MPNST are well known and include hypercellularity, remarkable cytological atypia, increased mitotic activity, and necrosis. ${ }^{4}$ Most MPNSTs are high grade and have a highly aggressive clinical course. However, in one large series, ${ }^{2} 18 \%$ of MPNSTs were reported as low grade. Other authors have described tumours that have exhibited perineural differentiation. ${ }^{5}$ Despite this variability there have been no systematic assessments of low grade MPNST.

The diagnosis of low grade MPNST is not easy. First, it is usually difficult to distinguish neurofibromas with varying degrees of atypia from MPNST. Second, the pathological features of low grade MPNST often overlap with those of other soft tissue tumours. Therefore, the purpose of our study was to describe the clinicopathological, immunohistochemical, and ultrastructural features of low grade MPNST and to discuss the main differential diagnoses.

\section{MATERIALS AND METHODS}

From our pathology files we retrieved surgical specimens from four patients in whom the diagnosis was low grade (World Health Organisation grade 1) MPNST (table 1). The tumours were analysed with respect to nuclear atypia, cellularity, nuclear enlargement, hyperchromasia, mitotic rate, and necrosis. For light microscopic study, all specimens were fixed in 10\% formalin and processed routinely for paraffin wax embedding. Sections ( $5 \mu \mathrm{m}$ thick) were stained with haematoxylin and eosin. The mitotic rate was expressed as the average mitotic count present in 10 high power fields, and 50 or more high power fields were examined in each case. Clinical data and follow up information were obtained from the hospital records, referring pathologists, and surgeons.

For immunohistochemical staining, the sections were dewaxed, rehydrated, and moistened with phosphate buffered saline $(\mathrm{pH} 7.4)$. The sections were pretreated in an autoclave at $121^{\circ} \mathrm{C}$ for 10 minutes in $10 \mathrm{mM}$ citrate buffer ( $\mathrm{pH}$ 6.0); they were then incubated with antibodies on an automated immunostaining system $\left(16000^{\mathrm{TM}}\right.$; BioGenex, San Ramon, California, USA) for 30 minutes at room temperature. Table 2 summarises the antibodies used in our study.

Electron microscopic examination was performed in one case (case 4). Suboptimal material was available from small fresh fragments of tissue; it was fixed in $2.5 \%$ glutaraldehyde, post-fixed in $1 \%$ osmium tetroxide, and embedded in epoxy resin. After treatment with uranyl acetate and lead citrate to create contrast, the ultrathin sections were examined under a transmission electron microscope.

\section{RESULTS}

\section{Clinical findings}

The ages of the patients at the time of surgery ranged from 32 to 72 years (mean, 58 years) (table 1). Two patients were male and two were female. The site of involvement was a

Abbreviations: MPNST, malignant peripheral nerve sheath tumour; NF1, neurofibromatosis type 1 
Table 1 Clinical findings of four low grade MPNSTs

\begin{tabular}{|c|c|c|c|c|c|c|c|c|}
\hline Case & NF1 & Age/Sex & Site & Symptom & Size $(\mathrm{cm})$ & Depth & Follow up & $\begin{array}{l}\text { Recurrence or } \\
\text { metastasis }\end{array}$ \\
\hline 1 & $P$ & $72 / M$ & Left groin & Mass & 3.5 & Superficial & 27 months & No \\
\hline 2 & $A$ & $32 / M$ & Left foot & Mass & 8 & Deep & 7 months & No \\
\hline 3 & A & $59 / \mathrm{F}$ & Right foot & Mass/pain & 3.5 & Deep & 3 months & No \\
\hline 4 & A & $71 / \mathrm{F}$ & Retroperitoneum & Free & 6.5 & Deep & 9 months & No \\
\hline
\end{tabular}

A, absent; MPNST, malignant peripheral nerve sheath tumour; NF1, neurofibromatosis type 1 ; $P$, present.

lower extremity in three patients and the retroperitoneum in one. Two of the four tumours occurred within, or were attached to, skeletal muscle, and one (case 1) was located in the dermal and subcutaneous tissues in a patient who had suffered from neurofibromatosis type l (NFl) since childhood. One patient (case 4) had been admitted for surgery for thyroid cancer, and a $6.5 \mathrm{~cm}$ diameter hypervascular mass had been found by chance in the retroperitoneum on routine radiological examination. All of the four patients had undergone radical surgical excision with no adjuvant treatment. The mean follow up period was 11 months (range, 327 months), and during this time no patients had developed recurrences or metastatic disease.

\section{Pathological features}

Grossly, the tumours were fusiform or oval and ranged from $3.5 \mathrm{~cm}$ to $8.0 \mathrm{~cm}$ (mean, 5.5 ) at their maximum dimension. Three of the four tumours appeared well circumscribed and were surrounded by thin, fibrous pseudocapsules, with a firm to hard consistency. One tumour (case 1), located within the dermis and subcutis, appeared as an ill defined solid mass. Many tiny nerve roots entered and exited the tumours. The cut surfaces appeared solid or myxoid and homogeneous, ranging from white to tan or yellow. No cystic degeneration, haemorrhage, or necrosis was seen.

Histologically, all tumours showed moderate hypercellularity, an increased nuclear to cytoplasmic ratio, and hyperchromasia. Slightly increased numbers of mitotic figures (fewer than 5/10 high power fields) were noted. Tumour necrosis was absent. The histological pattern varied from case to case.

The case 1 tumour occurred in association with NF1. This dermal and subcutaneous tumour contained interlacing bundles of collagen and a moderate amount of extracellular myxoid material (fig 1). Most of the tumour cells were long and spindle shaped and had slender, bipolar processes and wavy, dark staining nuclei. A few large and pleomorphic tumour cells were seen. These were not focal findings: all areas of the tumour showed the same histological pattern.

In case 2, the tumour was relatively hypocellular and composed of proliferations of plump to spindle shaped cells, with scant indistinct cytoplasm in a fibromyxoid background (fig 2). The spindle shaped cells had nuclei with a delicate chromatin pattern, inconspicuous eosinophilic nucleoli, and indistinct cell borders; the cells were arranged in a loose whorled or fascicular pattern. Nuclear pseudoinclusions were frequently noted. Scattered small blood vessels were seen among the tumour cells within the fibromyxoid stroma.

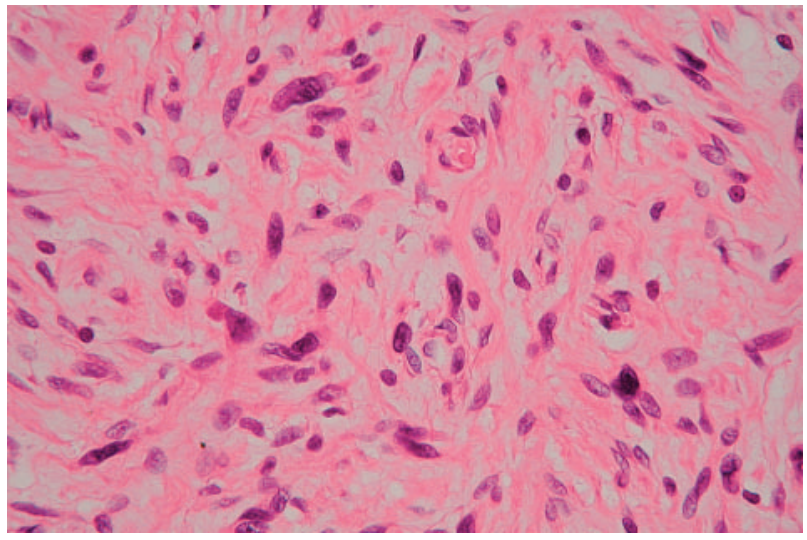

Figure 1 Tumour with increased cellularity and moderate nuclear atypia (case 1). The presence of dark smudged chromatin, nuclear enlargement, and abundant cytoplasm is noted (haematoxylin and eosin stained; original magnification, $\times 400$ ).

Table 2 Antibodies used in our study

\begin{tabular}{|c|c|c|c|}
\hline Antigen (clone; dilution) & Source & Pretreatment & Positive results \\
\hline $\begin{array}{l}\text { Vimentin (V9; } 1 / 200) \\
\text { S-100 protein }(1 / 2000)^{*} \\
\text { NSE (BBS/NC/VI-H14; } 1 / 200) \\
\text { CD57/Leu-7 (HNK-1; } 1 / 100) \\
\text { CD34 (my10; } 1 / 100) \\
\text { Desmin (D33; } 1 / 100) \\
\text { EMA (E29; } 1 / 100) \\
\text { Synaptophysin }(1 / 500)^{*} \\
\text { Chromogranin-A }(1 / 500)^{*} \\
\text { Factor VIII related antigen }(1 / 5000)^{*} \\
\text { MSA (HHF35; } 1 / 100) \\
\text { SMA (1A4; } 1 / 100) \\
\text { Cytokeratin }(A E 1 / 3 ; 1 / 100) \\
\text { gp100 protein (HMB-45; } 1 / 50) \\
\text { CD99/MIC2 (O-13; 1/50) } \\
\text { Ki-67 (MIB; } 1 / 100)\end{array}$ & $\begin{array}{l}\text { Dako Cytomation, Glostrup, Denmark } \\
\text { Dako Cytomation } \\
\text { Dako Cytomation } \\
\text { Becton Dickinson, San Jose, California, USA } \\
\text { Becton Dickinson } \\
\text { Dako Cytomation } \\
\text { Dako Cytomation } \\
\text { Dako Cytomation } \\
\text { Dako Cytomation } \\
\text { Dako Cytomation } \\
\text { Enzo, New York, USA } \\
\text { Dako Cytomation } \\
\text { Dako Cytomation } \\
\text { Dako Cytomation } \\
\text { Signet, Dedham, Massachusetts, USA } \\
\text { Dako Cytomation }\end{array}$ & $\begin{array}{l}\text { Autoclave } \\
\text { None } \\
\text { None } \\
\text { None } \\
\text { Autoclave } \\
\text { Autoclave } \\
\text { None } \\
\text { Autoclave } \\
\text { Autoclave } \\
\text { Autoclave } \\
\text { Autoclave } \\
\text { Autoclave } \\
\text { Autoclave } \\
\text { Autoclave } \\
\text { Autoclave } \\
\text { Autoclave }\end{array}$ & $\begin{array}{l}4 / 4 \\
4 / 4 \\
2 / 4 \\
1 / 4 \\
1 / 4 \\
0 / 4 \\
0 / 4 \\
0 / 4 \\
0 / 4 \\
0 / 4 \\
0 / 4 \\
0 / 4 \\
0 / 4 \\
0 / 4 \\
0 / 4 \\
0-1 \%(1 / 4) \\
1-5 \%(3 / 4)\end{array}$ \\
\hline
\end{tabular}

*Polyclonal antibody.

EMA, epithelial membrane antigen; MSA, muscle specific actin; NSE, neurone specific enolase; SMA, smooth muscle actin. 


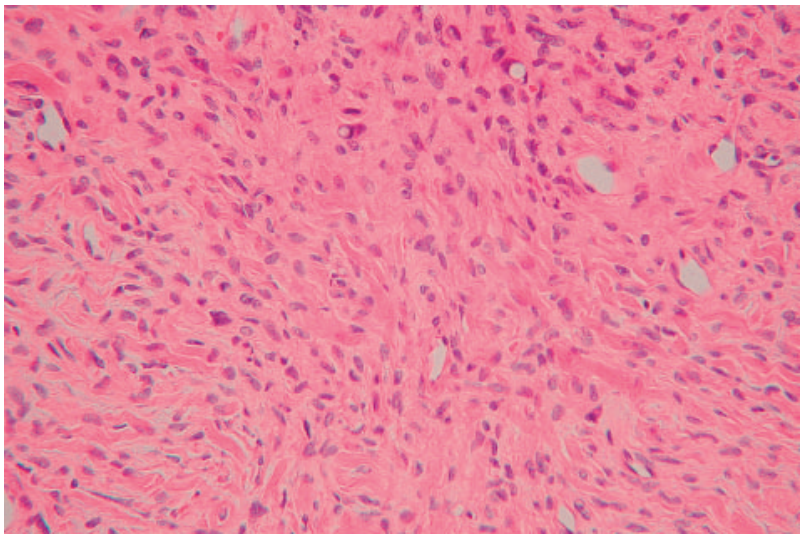

Figure 2 Neoplastic cells of a low grade fibromyxoid sarcoma-like malignant peripheral nerve sheath tumour are arranged in a loose whorled or fascicular pattern within a fibromyxoid matrix (case 2). Nuclear pseudoinclusions and small blood vessels are seen (haematoxylin and eosin stained; original magnification, $\times 200$ ).

In case 3, the tumour consisted mostly of epithelioid cells (fig 3). The epithelioid cells were mainly ovoid, contained abundant pink cytoplasm, were arranged in compact sheets, and had clear nuclei with relatively conspicuous nucleoli. Conventional growth patterns, characterised by alternating loose and dense areas of short spindle shaped cells and increased perivascular cellularity, were observed in some areas of the tumour (fig 4).

In case 4 , the tumour exhibited a patternless architecture and a haemangiopericytoma-like arrangement, consisting of many blood vessels (fig 5). The walls of the blood vessels were lined with a single layer of flattened endothelium, and perivascular hyalinisation was occasionally seen. The tumour cells were rounded or short and spindle shaped, with prominent eosinophilic cytoplasm and distinct cytoplasmic borders, and were intermixed with collagen bundles (fig 6). Small numbers of large pleomorphic cells with occasional nuclear pseudoinclusions were observed. Lymphocytic infiltration was also present. These findings were noted in all areas of the tumour.

\section{Immunohistochemical studies}

In all tumours, immunoreactivity for both S-100 protein and vimentin was strong and diffuse (fig 7). Focal positive staining for neurone specific enolase and CD57/Leu-7 was seen in one tumour (case 4), and positivity for neurone specific enolase alone was present in another (case 3). In one tumour (case 2 ), only certain areas of the cells were positive for CD34, and the areas that stained were often the slender and elongated cytoplasmic processes of the tumour cells. Ki67 (MIB-1) nuclear staining in the four tumours ranged from $1 \%$ to $5 \%$ (mean, $3.8 \%$ ). There was no immunoreactivity with antibodies to epithelial membrane antigen, desmin, synaptophysin, chromogranin-A, factor VIII related antigen, muscle specific actin, $\alpha$ smooth muscle actin, cytokeratin, gpl00 protein, or CD99.

\section{Electron microscopic findings}

Ultrastructurally, the tumour cells had mesenchymal morphology, with untapered, branching cytoplasmic processes, surrounded by a basal lamina (fig 8). The nuclei were elongated or ovoid, and had abundant euchromatin with clumpy heterochromatin. Nucleoli were often inconspicuous. The cytoplasm was abundant and contained scattered ribosomes, polyribosomes, rough endoplasmic reticulum, many intermediate filaments, and occasional microtubules. The cell processes were joined by rudimentary cell junctions

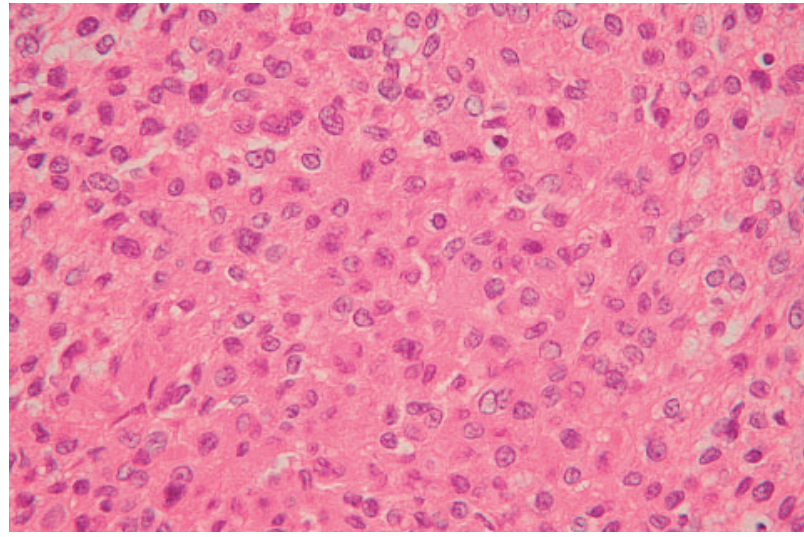

Figure 3 A low grade epithelioid malignant peripheral nerve sheath tumour (case 3) showing compact sheets of epithelioid cells with clear nuclei and abundant pink cytoplasm (haematoxylin and eosin stained; original magnification, $\times 400$ ).

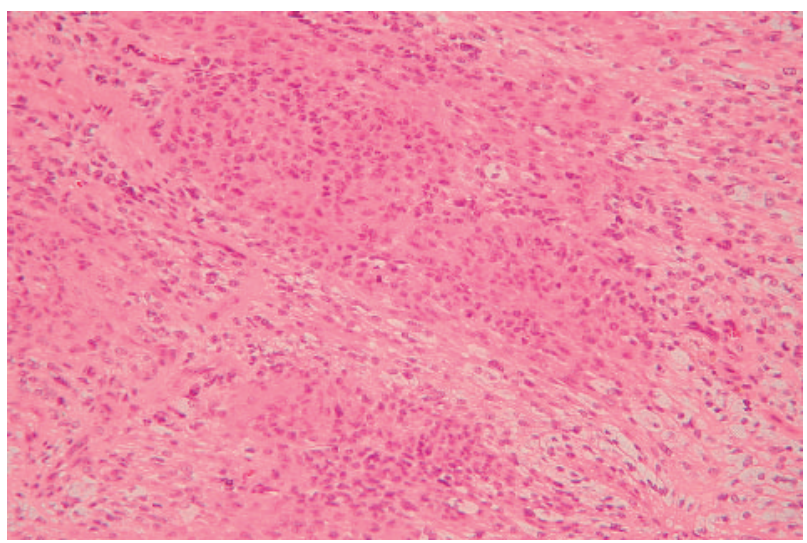

Figure 4 Some areas of this tumour (case 3) display an alternating loose and densely cellular pattern of short spindle shaped cells (haematoxylin and eosin stained; original magnification, $\times 200$ ).

and bordered by a discontinuous external basal lamina, along with occasional long spacing collagen. Electron dense cell membrane plaques and a few pinocytotic vesicles were also noted on the cell surfaces. These findings proved that the tumour cells bore the features of Schwann cells.

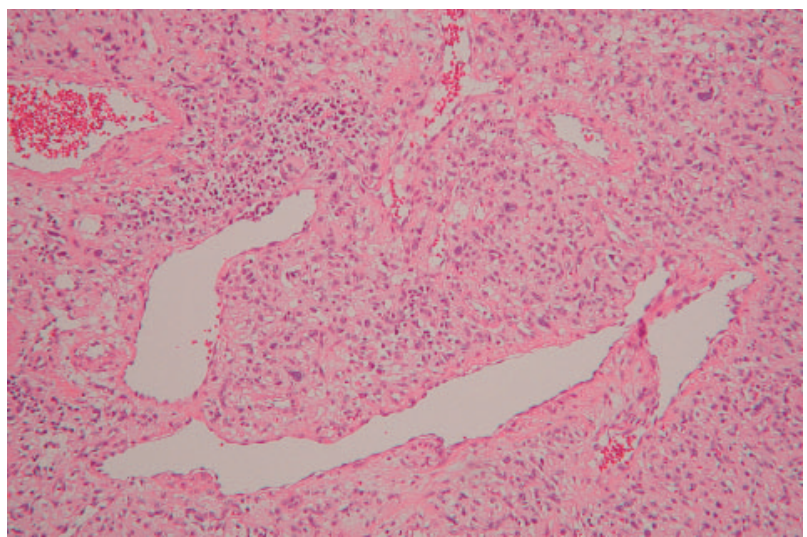

Figure 5 Low power view of a low grade haemangiopericytoma-like malignant peripheral nerve sheath tumour with branching vascular structures (case 4). Lymphocytic infiltration is frequent (haematoxylin and eosin stained; original magnification, $\times 100$ ). 


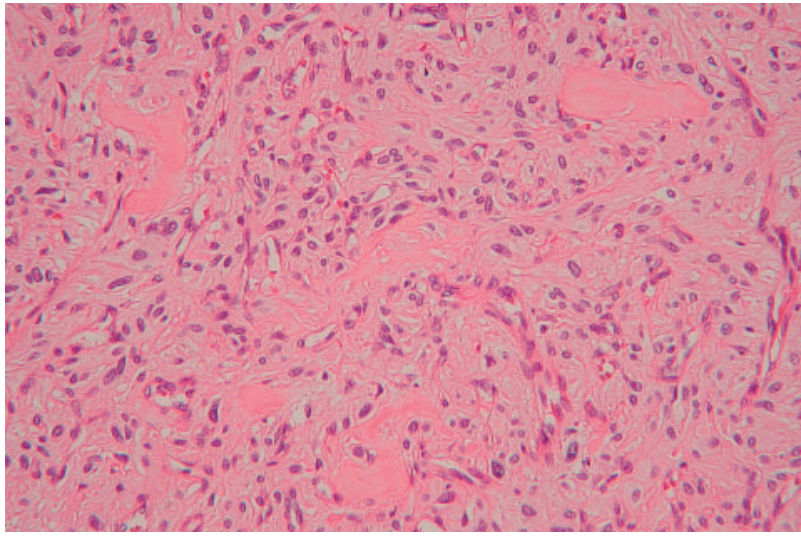

Figure 6 High power view of this fumour (case 4) with a haphazard arrangement of short spindle shaped cells around small vessels, and interstitial hyalinised collagen (haematoxylin and eosin stained; original magnification, $\times 200$ ).

\section{DISCUSSION}

In recent years there has been increasing consensus in the diagnosis of MPNST. Immunohistochemical and ultrastructural analysis plays an important role in accurate diagnosis, particularly if the tumour is not associated with NFl or has no continuity with a nerve. S- 100 protein is the antigen most widely used for neural differentiation, and between 30\% and $67 \%$ of MPNSTs stain positively for S-100 protein to some extent. $^{6-8}$ It has been reported that low grade MPNSTs show diffuse and intense staining for S-100 protein, as found in our study. ${ }^{9}$ It is thought that most low grade MPNSTs arise in neurofibromas. ${ }^{9}$ In this situation, the histological criteria that enable a positive diagnosis of low-grade MPNST are the presence of hypercellularity, general nuclear enlargement, mitoses, and hyperchromasia, as described previously. ${ }^{9}$ Hirose et al described low grade MPNSTs that had perineural cell differentiation and showed immunoreactivity for epithelial membrane antigen and a lack of S-100 protein staining. ${ }^{5}$ However, our four cases were conventional MPNSTs. They exhibited variability in their cytology and histological patterns, and needed to be differentiated from other soft tissue tumours.

The case 1 tumour would most likely be confused with atypical neurofibroma. Our diagnosis was confirmed by the fact that uniform nuclear enlargement and hyperchromasia were consistently seen, and the cellularity was extreme. In contrast, neurofibroma with atypical features usually shows focal or absent nuclear enlargement and hyperchromasia, and often has less extensive hypercellularity.

The case 2 tumour showed low grade fibromyxoid sarcoma-like features, and would need to be differentiated from the spindle cell sarcomas, including low grade fibromyxoid sarcoma, low grade myxofibrosarcoma, and fibrosarcoma. Histologically, this case closely resembled low grade fibromyxoid sarcoma, with spindle cells and the presence of a fibromyxoid stroma. However, low grade fibromyxoid sarcoma exhibits a predominantly whorled arrangement of cells, and has a fibroblastic nature ${ }^{10}{ }^{11}$ and a lack of S-100 protein immunoreactivity. ${ }^{11}$ Low grade myxofibrosarcomas are characterised by fibroblastic and histiocytic cells; they are uniformly myxoid and lack areas with a fibrous stroma. Fibrosarcoma is often composed of myxoid stroma and may contain thick bundles of collagen, but it differs from low grade MPNST because it has long, sweeping fascicles or a herringbone pattern, a more prominent myxoid matrix, and greater hyperchromasia.

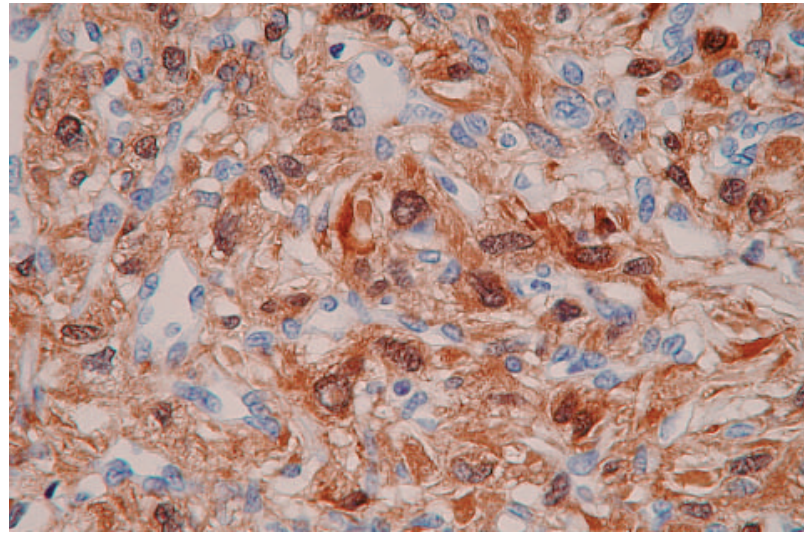

Figure 7 A low grade haemangiopericytoma-like malignant peripheral nerve sheath tumour (case 4) shows striking nuclear and cytoplasmic immunoreactivity for S-100 protein (original magnification, $\times 400$ ).

Approximately 5\% of MPNSTs are either partly or purely epithelioid. ${ }^{12-14}$ Although reported examples of epithelioid MPNST are often high grade, the case 3 tumour had a low grade epithelioid appearance. The diagnosis depended upon the fact that the tumour arose from a tiny nerve root; moreover, the tumour cells had a spindle cell component typical of MPNST and were positive for S-100 protein. These findings made the differential diagnosis easier from among metastatic carcinoma, sweat gland carcinoma, and malignant melanoma, as described previously. ${ }^{12}$

Information on haemangiopericytoma-like MPNST is limited. It has been reported that nine of 37 cases of malignant schwannoma showed a partial haemangiopericytoma-like pattern. ${ }^{15}$ However, our case showed a purely haemangiopericytoma-like pattern, so perhaps this is the first such documented case. Solitary fibrous tumour, haemangiopericytoma, and pleomorphic hyalinising angiectatic tumour arise in the differential diagnosis. Solitary fibrous tumour and haemangiopericytoma are tumours that contain spindle shaped cells and show a haemangiopericytoma-like branching vascular pattern. Both can occur in the retroperitoneum. Unlike our case, they usually demonstrate features of fibroblastic and pericytic differentiation, and show immunoreactivity for CD34 and CD99, and a lack of S-100 protein

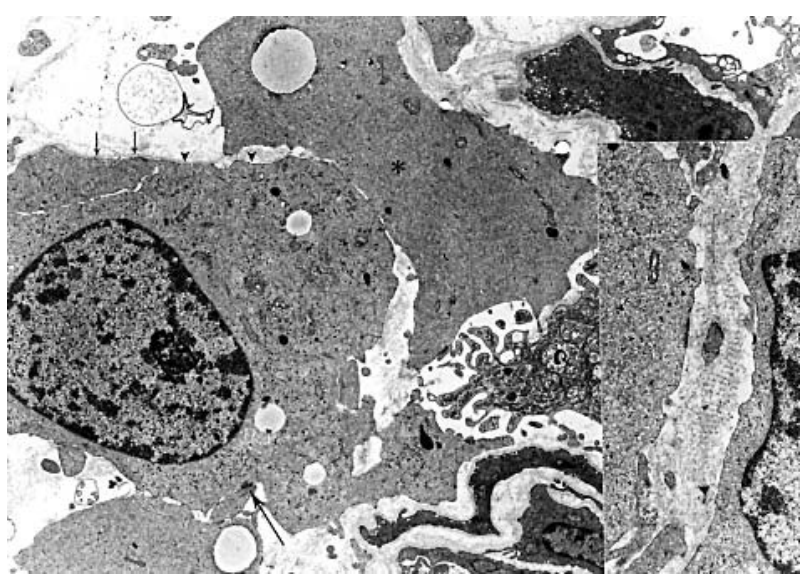

Figure 8 An electron micrograph of low grade haemangiopericytomalike malignant peripheral nerve sheath tumour (case 4). Tumour cells are occasionally connected by rudimentary cell junctions (large arrow). Basal lamina (small arrows) and a small number of pinocytotic vesicles (arrowheads) are seen on the cell surface. Occasional microtubules (asterisk) are evident in the cytoplasm. Inset: Long spacing collagen (original magnification, $\times 10000$ ). 


\section{Take home messages}

- We describe four cases of low grade malignant peripheral nerve sheath tumour (MPNST) that exhibited variability in cytology and histological pattern

- Careful clinical and histological evaluation, together with S-100 protein immunostaining and ultrastructural analysis, are essential for the accurate diagnosis of low grade MPNST

immunostaining. ${ }^{16-20}$ Pleomorphic hyalinising angiectatic tumour is a recently described entity that is characterised by oval to spindle shaped and pleomorphic cells, and clusters of ectatic vessels with perivascular hyalinisation. ${ }^{21}$ It differs from our case because of its various proliferating patterns, more fascicular pattern, and lack of staining for S-100 protein. $^{22}$

"Our case showed a purely haemangiopericytoma-like pattern, so perhaps this is the first such documented case"

MPNST has a highly aggressive clinical behaviour. About $60 \%$ of patients die of their disease, and the five and 10 year survival rates are $34 \%$ and $23 \%$, respectively. ${ }^{2}$ No significant correlation has been noted between survival and histological grade. ${ }^{2}$ Although the clinical follow up of our patients was short, no recurrences or metastases have been detected since radical surgical excision. Nevertheless, close follow up is required.

In summary, we have presented four cases of low grade MPNST that exhibited variability in cytology and histological pattern. Each tumour showed immunoreactivity for S-100 protein. Our findings suggest that careful clinical and histological evaluations, along with S-100 protein immunostaining, are essential for accurate diagnosis.

\section{ACKNOWLEDGEMENTS}

This work was supported in part by a Grant-in-Aid for Cancer Research from the Ministry of Health, Labor and Welfare, Japan.

\section{Authors' affiliations}

U Yamaguchi, H Chuman, A Kawai, Y Ito, Y Beppu, Division of Orthopaedic Oncology, National Cancer Centre, Hospital and Research Institute, Tokyo 104-0045, Japan

T Hasegawa, Division of Pathology, National Cancer Centre, Hospital and Research Institute

T Hirose, Department of Pathology, Saitama Medical School, Saitama 350-0495, Japan

\section{REFERENCES}

1 Lewis JJ, Brennan MR. Soft tissue sarcomas. Curr Probl Surg 1996;33:817-72.

2 Ducatman BS, Scheithauer BW, Piepgras DG, et al. Malignant peripheral nerve sheath tumor. A clinicopathologic study of 120 cases. Cancer 1986:57:2006-21.

3 Hruban RH, Shiu MH, Senie RT, et al. Malignant peripheral nerve sheath tumors of the buttock and lower extremity. A study of 43 cases. Cancer 1990;66:1253-65.

4 Weiss SW, Goldblum JR. Malignant tumors of the peripheral nerves. In: Enzinger and Weiss's soft tissue tumors, 4th ed. St Louis: Mosby, 2001:1209-63.

5 Hirose T, Scheithaver BW, Sano T. Perineural malignant peripheral nerve sheath tumor (MPNST). A clinicopathologic, immunohistochemical, and ultrastructural study of seven cases. Am J Surg Pathol 1998;22:1368-78.

6 Matsunou H, Shimoda T, Kamimoto S, et al. Histopathologic and immunohistochemical study of malignant tumors of peripheral nerve sheath (malignant schwannoma). Cancer 1985;56:2269-79.

7 Weiss SW, Langloss JM, Enzinger FM. Value of S-100 protein in the diagnosis of soft tissue tumors with particular reference to benign and malignant Schwann cell tumors. Lab Invest 1983;49:299-308.

8 Wick MR, Swanson PE, Scheithaver BW. Malignant peripheral nerve sheath tumor: an immunohistochemical study of 62 cases. Am J Clin Pathol 1987;87:425-33.

9 Scheithauer BW, Woodruff JM, Erlandson RA. Primary malignant tumors of peripheral nerve. Atlas of tumor pathology: tumors of the peripheral nervous system, 3rd series, Fascicle 24. Washington: Armed Forces Institute of Pathology, 1999:303-72.

10 Evans HL. Low grade fibromyxoid sarcoma. Am J Surg Pathol 1993; 17:595-600.

11 Goodlad JR, Mentzel T, Fletcher CDM. Low grade fibromyxoid sarcoma: clinicopathological analysis of eleven new cases in support of a distinct entity. Histopathology 1995;26:229-37.

12 DiCarlo EF, Woodruff JM, Bansal M, et al. The purely epithelioid malignant peripheral nerve sheath tumor. Am J Surg Pathol 1986;10:478-90.

13 Laskin WB, Weiss SW, Bratthaver GL. Epithelioid variant of malignant peripheral nerve sheath fumor (malignant epithelioid schwannoma). Am J Surg Pathol 1991;15:1136-45.

14 Lodding P, Kindblom LG, Angervall L. Epithelioid malignant schwannoma. Virchows Arch A Pathol Anat Histopathol 1986;409:433-51.

15 Tsuneyoshi M, Daimaru Y, Enjoji M. Malignant hemangiopericytoma-like and other sarcomas with hemangiopericytoma-like pattern. Pathol Res Pract 1984;178:446-53.

16 Burnnemann RB, Ro JY, Ordonez NG, et al. Extrapleural solitary fibrous tumor: a clinicopathologic study of 24 cases. Mod Pathol 1999;12:1034-42.

17 Mentzel T, Bainbridge TC, Katenkamp D. Solitary fibrous tumor: clinicopathological, immunohistochemical, and ultrastructural analysis of 12 cases arising in soft tissues, nasal cavity and prostate. Virchows Arch 1997;430:445-53.

18 Renshaw AA. O13 (CD99) in spindle cell tumors. Reactivity with hemangiopericytoma, solitary fibrous tumor, synovial sarcoma, and meningioma but rarely with sarcomatoid mesothelioma. Appl Immunohistochem 1995;3:250-6.

19 Suster S, Nascimento AG, Miettinen M, et al. Solitary fibrous tumors of soft tissue. A clinicopathologic and immunohistochemical study of 12 cases. Am J Surg Pathol 1995; 19:1257-66.

20 Hasegawa T, Hirose T, Seki K, et al. Solitary fibrous tumor of the soft tissue. An immunohistochemical and ultrastructural study. Am J Clin Pathol 1996; 106:325-31

21 Smith MEF, Fisher C, Weiss SW. Pleomorphic hyalinizing angiectatic tumor of soft parts: a low-grade neoplasm resembling neurilemoma. Am J Surg Pathol 1996;20:21-9.

22 Fukunaga $M$, Ushigome S. Pleomorphic hyalinizing angiectatic tumor of soft parts. Pathol Int 1997;47:784-8. 